\title{
Changes in Umbilical and Cerebral Blood Flow in Pregnancies Diagnosed with Clinical Chorioamnionitis: Is Chorioamnionitis Predictable?
}

\author{
Berfin OKMEN OZKAN', Emre EKMEKCI ${ }^{2}$ \\ Sanliurfa,Turkey
}

\begin{abstract}
OBJECTIVE: To evaluate the predictability of clinical chorioamnionitis by Doppler changes in fetal middle cerebral and umbilical arteries, at hospitalized patients due to preterm premature rupture of membranes.

STUDY DESIGN: Patients who were admitted and hospitalized due to preterm premature rupture of membranes between 24 weeks and 33 weeks and 6 days pregnancies are included in the study. Demographic data of patients, gestational age at referring to hospitalization, fetal presentation at admission, delivery time, delivery indications and total follow-up time until delivery of each case were recorded.

RESULTS: A total of 108 patients were evaluated retrospectively. The rate of clinical chorioamnionitis was $5.55 \%$ (6/108). There was no significant difference between pregnancies terminated with the diagnosis of clinical chorioamnionitis and pregnancies terminated with other indications in terms of the maximum systolic velocity at the middle cerebral artery and umbilical artery pulsatility indices.

CONCLUSION: Clinical chorioamnionitis is a serious complication and is more common in patients being followed up after preterm premature rupture of membranes. Although it is crucial to be predicted due to neonatal worse prognosis, it cannot be predicted by middle cerebral and umbilical artery Doppler evaluation.
\end{abstract}

Keywords: Chorioamnionitis, Middle cerebral artery, Preterm premature rupture of membranes, Umbilical artery

Gynecol Obstet Reprod Med 2019;25(3):123-127

\section{Introduction}

Chorioamnionitis is a serious complication of pregnancy that is caused by bacterial infection or inflammation of the fetal amnion and chorionic membranes have been shown to be

${ }^{1}$ Sanliurfa Education and Research Hospital Obstetrics and Gynecology Department Sanliurfa, Turkey.

${ }^{2}$ Sanliurfa Education and Research Hospital Obstetrics and Gynecology Department Perinatology Clinic, Sanliurfa, Turkey.

Address of Correspondence: Berfin Okmen Ozkan Sanliurfa Education and Research Hospital Obstetrics and Gynecology Department, 63200 Sanliurfa, Turkey berfin.okmen@hotmail.com

Submitted for Publication: 30.11.2019

Revised for: Publication:

25.01.2019

21.02.2019

Accepted for Publication:

BOO:0000-0003-3707-2270,

EE: 0000-0003-2494-3073

\begin{tabular}{|c|c|}
\hline Quick Response Code: & Access this article online \\
\cline { 2 - 2 } & $\begin{array}{c}\text { Website: www.gorm.com.tr } \\
\text { e- mail: info@gorm.com.tr }\end{array}$ \\
\cline { 2 - 3 } & DOI:10.21613/GORM.2018.863 \\
\hline
\end{tabular}

How to cite this article: Okmen Ozkan B. end Ekmekci E. Changes in Umbilical and Cerebral Blood Flow in Pregnancies Diagnosed with Clinical Chorioamnionitis. Is Chorioamnionitis Predictable? Gynecol Obstet Reprod Med. 2019;25(3):123-127 commonly associated with preterm premature rupture of membranes (PPROM) (1). Intra-amniotic infection is common in PPROM patients. Histological confirmation of chorioamnionitis is being detected in approximately $50-60 \%$ of PPROM patients and is inversely correlated with gestational age at birth $(2,3)$. If the characteristic clinical signs and symptoms; maternal fever, leukocytosis, fetal/maternal tachycardia, uterine tenderness and definite purulent fluid from the cervical os are present, the condition is described as clinical chorioamnionitis. Clinical chorioamnionitis is usually diagnosed during attempts to delay the preterm delivery especially before 30 weeks of gestation or with PPROM. Also, microorganisms are more virulent at clinical chorioamnionitis compared to histological chorioamnionitis $(4,5)$.

Recent epidemiologic and clinical investigations on neonatal outcomes of pregnancies complicated with clinical chorioamnionitis indicate that newborn is at increased risk of developing intraventricular hemorrhage, periventricular leucomalacia and cerebral palsy (6-8).

Increased peak and end diastolic velocities at middle and anterior cerebral arteries are reported at newborns with early- 
onset neonatal sepsis. Also velocities were higher in the case of sepsis-associated encephalopathy at newborns compared to group with neonatal sepsis and no encephalopathy (9).

Prediction of chorioamnionitis will be beneficial during the follow-up of patients after PPROM due to the association of chorioamnionitis with worse neonatal prognosis. In this study, we evaluated the utility of middle cerebral artery (MCA) peak systolic velocities in the prediction of chorioamnionitis during follow-up of PPROM patients. Also, the umbilical artery (UA) Doppler is evaluated in the same patient group.

\section{Material and Method}

This retrospective cohort study is conducted in the Department of Obstetrics and Gynecology, Maternal-Fetal Medicine Unit, Sanliurfa Education and Research Hospital, Sanliurfa, Turkey. Retrospectively collected data is acquired from patients' medical records who have been hospitalized with a diagnosis of PPROM during 24-34 weeks of gestation, between September 2017 and October 2018. The written consent is taken from all patients at admission to hospital about the use of their medical data in clinical researches. The study design was in accordance with the Helsinki Declaration (Association 2014). Approval and permission for the study about the provision of patient data are taken from an institutional clinical board (registration number: 96537014-003876). The unit is a busy tertiary center in the east of Turkey getting referral patients from the region with about 40000 deliveries in a year.

The patients included in this study are all inpatients and all are followed in the clinic. If delivery started spontaneously in the first 24 hours after admission for the rupture of membranes, it is described as preterm delivery and not included in the study. Patients who delivered due to other obstetrical indications not related to PPROM and its complications, such as severe preeclampsia or abnormal obstetrical Doppler indices at the very beginning of the study were not included in the study. Multiple pregnancies, pregnancies complicated with intra uterine growth retardation, preeclampsia, and indirect Coombs positive patients were not included in the study.

Antibiotic prophylaxis was started to all hospitalized patients at admission (azithromycine 1gr once and ampicillin $1 \mathrm{~g}$ intravenously every 6 hours for 7 days). All PPROM patients are being evaluated with ultrasonography including umbilical and MCA Doppler, during admission to our clinic. Doppler evaluations were repeated in three days. Diagnosis of clinical chorioamnionitis is made according to the presence of fever $\left(>38.0^{\circ} \mathrm{C}\right)$ and two or more of the following: maternal and fetal tachycardia, uterine tenderness, foul-smelling amniotic fluid (vaginal discharge), and maternal leukocytosis (10). MCA peak systolic velocities (PSV) and UA pulsatility indices of exams are recorded at admission and the last one prior to delivery. Ultrasound exams are performed by the same physician (Ekmekci E). MCA-PSV measurements were standardized as previously described (11). Ultrasonographic measurements were performed by Voluson P8 (General Electric, Milwaukee, WI, USA). The Doppler gate was placed at the proximal third of the MCA from its origin(internal carotid artery), with an angle of insonation of less than $15^{\circ}$. PSV was expressed as centimeters per second and corresponded to the highest value out of 2-3 consecutive measures. Each MCA Doppler measurement was interpreted as multiples of the median (MoM) for corresponding gestational age. The UA Doppler waveforms were recorded from free loop of cord.

Demographic data of patients, gestational age at referring to hospitalization, fetal presentation at admission, delivery time, delivery indications and total follow-up time until delivery of each case were recorded. Indirect Coombs results were recorded to exclude the patients with fetal erythrocyte alloimmunization. The clinical chorioamnionitis rate was calculated in study group. MCA PSV MoM rates and pulsatility indices are compared between chorioamnionitis patients and patients without the diagnosis of clinical chorioamnionitis.

Statistical analyses were performed using MedCalc Statistical Software (MedCalc Software, Ostend, Belgium). Statistical data were reported descriptively due to small number of chorioamnionitis cases. Categorical variables were given as median (minimum-maximum). Independent samples t-test was used to assess the differences between groups. $P<$ 0.05 was considered statistically significant.

\section{Results}

A total of 117 patients' medical records were evaluated retrospectively who were admitted and followed in the clinic until delivery. Indirect Coombs was negative in all patients. Three patients delivered due to abnormal UA Doppler (reverse or absent end-diastolic flow) and fetal growth retardation. Two patients delivered due to severe preeclampsia and they were excluded from the study. Totally, 108 patients are included in this study.

The mean maternal age was $28 \pm 6.9$ years. Median gravida was three (1-12). The mean gestational age at diagnosis of PPROM was $29 \pm 2.6$ weeks. Total of 6 clinical chorioamnionitis is diagnosed during the study $(5.55 \%)$ and those were delivered afterwards because of chorioamnionitis. The other 102 cases were delivered due to other indications (preterm labor, cord prolapse, abruptio placenta, and fetal distress). The mean follow-up period until delivery was $10.5 \pm 4.7$ days (2112 days) in non-chorioamnionitis group. Clinical chorioamnionitis is diagnosed in the first 24 hours after membrane rupture in two patients. Diagnosis of clinical chorioamnionitis is made during follow-up at four patients (6-28 days).

Mean MCA PSV was $0.93 \pm 0.15 \mathrm{MoM}$ at non-chorioam- 
nionitis group (0.65-1.37 MoM) at admission to clinic while that was $0.92 \pm 0.1 \mathrm{MoM}$ at chorioamnionitis group (0.84-1.12 MoM $)(p=0.8648)$. Mean UA pulsatility indices were $0.96 \pm 0.22$ at non-chorioamnionitis group (0.53-1.53) at admission to clinic while that was $0.99 \pm 0.16 \mathrm{MoM}$ at chorioamnionitis group (0.72-1.21), $(\mathrm{p}=0.9699)$. Distribution of values in each group is reported separately in Box and Whisker Plots (Figure 1-4).

All babies were delivered just after the diagnosis of clinical chorioamnionitis and no neonatal sepsis occurred.

\section{Discussion}

Chorioamnionitis is one of the most unfavorable complications for clinicians due to its relation with maternal and fetal poor outcomes during follow-up of patients with PPROM. We aimed to evaluate the utility of fetal Doppler ultrasound to predict chorioamnionitis during follow-up of PPROM patients and our findings do not indicate any association with fetal Doppler ultrasound findings and clinical chorioamnionitis.

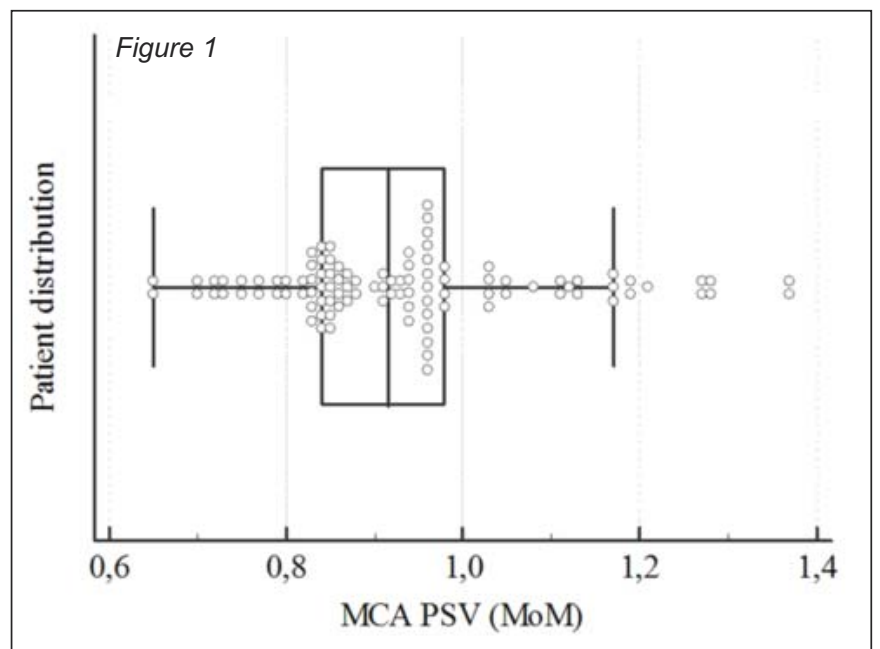

The rates reported about the clinical chorioamnionitis rates during conservative management of pregnancies with PPROM are variable. A study from India is reported as $6.6 \%$ on 150 patients (12). Clinical chorioamnionitis rate was $5.55 \%(6 / 108)$ in our study. However, the two patients with chorioamnionitis were diagnosed in the first 24 hours after membrane rupture. They may not be grouped the same with others because the chorioamnionitis was present at admission and not diagnosed on follow-up after membrane rupture. It's more accurate not to describe chorioamnionitis as a result of membrane rupture for these patients. On the contrary, it would be more accurate to define the membrane rupture as a result of chorioamnionitis for them. The presence of intra-amniotic infection after spontaneous membrane rupture is reported variously in different studies (13-15). And the rates are higher in earlier gestational ages. Pintucci et al. reported $2.3 \%$ clinical chorioamnionitis rate at term premature rupture of membranes (16). The rates are higher with lower gestational ages. Histological chorioamnionitis is being detected higher than clinical chorioamnionitis. It's reported about $50-60 \%$ in various studies among PPROM patients before 34. gestational

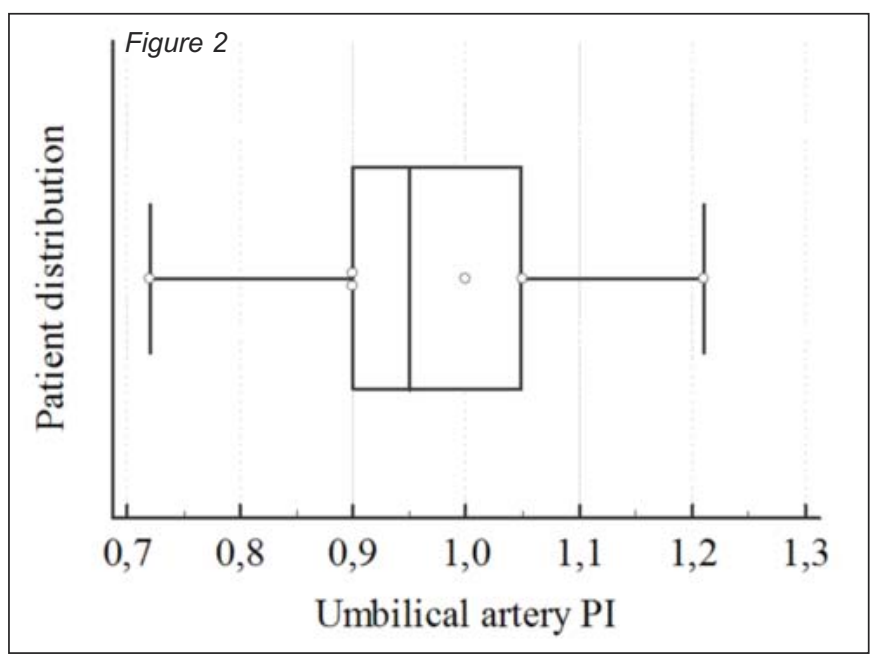

Figure 1 and 2: Distribution of middle cerebral artery peak stctolic velocities values in chorioamnionitis positive and negative groups
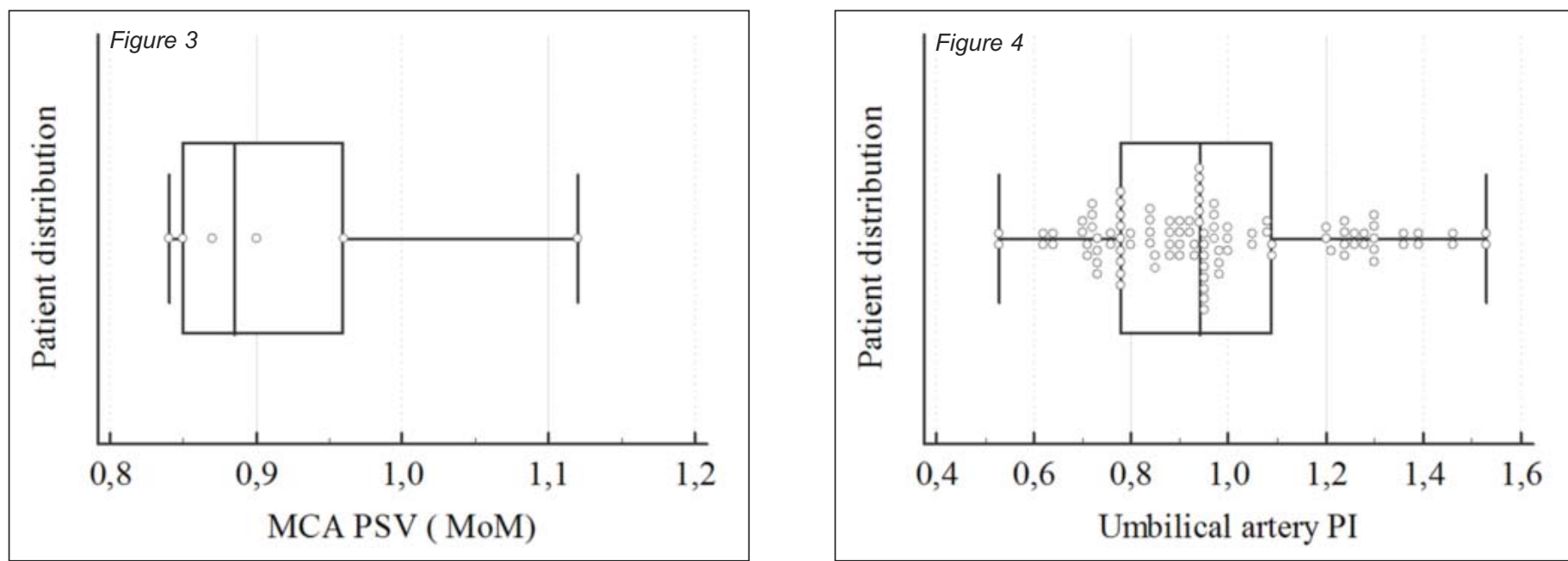

Figure 3 and 4: Distribution of umbilical artery PI values in chorioamnionitis positive and negative groups 
week $(17,18)$. However, clinical chorioamnionitis rate is reported as $6.6 \%$ by Minakeshi et al. (12). Higher rates with increasing prematurity remarks the role of intra-amniotic infection at preterm labor.

Shimy et al reported higher blood flow velocities in cerebral arteries at newborns with neonatal sepsis and was associated with the severity of the disease (9). Fetal inflammatory response may be associated with higher velocities in blood flow that is remarked at newborns with neonatal sepsis but this association is not detected at clinical chorioamnionitis patients' fetuses. The time at diagnosis of clinical chorioamnionitis may be early to develop a fetal response in circulation. No change in blood flow at prenatal Doppler studies may be explained by this.

Various studies have been made to predict chorioamnionitis on the follow-up of PPROM patients. But almost all of these are based on the diagnosis of histological chorioamnionitis. Cho HY reported that the delta neutrophil index could be a predictive marker for histological chorioamnionitis in patients with PPROM (19). Pino MD et al did not detect any change on fetal Doppler parameters in 41 women with PPROM who subsequently developed clinical chorioamnionitis (20). Similarly, our findings were consistent with them; we did not detect any change on MCA and UA Doppler on patients with PPROM who developed chorioamnionitis on follow-up. Although the main purpose of our study was to analyze fetal cerebral blood flow changes at chorioamnionitis, we analyzed fetal systemic blood flow by umbilical artery Doppler indices.

The major strength of this study is that this is the first study evaluating the efficacy of MCA and umbilical artery Doppler changes on PPROM patients about the prediction of chorioamnionitis in a large study population. Some limitations should be mentioned. Diagnosis of clinical chorioamnionitis is not confirmed histopathologically because of the retrospective design of the study. Also, histopathologic chorioamnionitis rate is not detected in the study.

In conclusion, clinical chorioamnionitis is a serious and feared complication of PPROM during follow-up. Clinical chorioamnionitis rate is not as high as to be reluctant to conservative management of PPROM patients. However, delivery should be considered at the earliest suspicion about clinical chorioamnionitis. Still, there is no proven method to predict clinical chorioamnionitis prior to the clinical signs of that.

\section{Acknowledgments: None}

Source of funding: None

Conflict of interests: The authors declare that they have no conflict of interests.

Author Contributions: BOO: Data collection, Analysis and interpretation, Writer. EE: Supervision, Concept, Design and processing, Statistical analysis and Data interpretation.

\section{References}

1. Garite TJ, Freeman RK. Chorioamnionitis in the preterm gestation. Obstet Gynecol. 1982;59(5):539-45.

2. Park KH, Kim SN, Oh KJ, Lee SY, Jeong EH, Ryu A. Noninvasive prediction of intra-amniotic infection and/or inflammation in preterm premature rupture of membranes. Reprod Sci. 2012;19(6):658-65.

3. Elimian A, Verma U, Beneck D, Cipriano R, Visintainer $\mathrm{P}$, Tejani N. Histologic chorioamnionitis, antenatal steroids, and perinatal outcomes. Obstet Gynecol. 2000;96(3):333-6.

4. Jobe AH, Kallapur S, Moss TJ. Inflammation/infection: effects on the fetal/newborn lung. The newborn lung: Elsevier; 2008. p. 119-40.

5. Holzman C, Lin X, Senagore P, Chung H. Histologic chorioamnionitis and preterm delivery. Am J Epidemiol. 2007;166(7):786-94.

6. De Felice C, Toti P, Laurini RN, Stumpo M, Picciolini E, Todros T, et al. Early neonatal brain injury in histologic chorioamnionitis. J Pediatr. 2001;138(1): 101-4.

7. Dammann O, Leviton A. Maternal intrauterine infection, cytokines, and brain damage in the preterm newborn. Pediatr Res. 1997;42(1):1-8.

8. Zupan V, Gonzalez P, Lacaze Masmonteil T, Boithias C, D'Allest AM, Dehan M, et al. Periventricular leukomalacia: risk factors revisited. Dev Med Child Neurol. 1996;38(12):1061-7.

9. El Shimy MS, El-Raggal NM, El-Farrash RA, Shaaban HA, Mohamed HE, Barakat NM, et al. Cerebral blood flow and serum neuron-specific enolase in early-onset neonatal sepsis. Pediatr Res. 2018;84:261-6.

10. Higgins RD, Saade G, Polin RA, Grobman WA, Buhimschi IA, Watterberg K, et al. Evaluation and management of women and newborns with a maternal diagnosis of chorioamnionitis: summary of a workshop. Obstet Gynecol. 2016;127(3):426-36.

11. Friszer S, Maisonneuve E, Macé G, Castaigne V, Cortey A, Mailloux A, et al. Determination of optimal timing of serial in utero transfusions in red-cell alloimmunization. Ultrasound Obstet Gynecol. 2015;46(5):600-5.

12. Rana M, Patra S, Puri M, Trivedi SS. Fetomaternal outcome in preterm premature rupture of membrane. International Journal of Infertility and Fetal Medicine. 2014;5(1):18-21.

13. Cahill AG, Duffy CR, Odibo AO, Roehl KA, Zhao Q, Macones GA. Number of cervical examinations and risk of intrapartum maternal fever. Obstet Gynecol. 2012;119 (6):1096-101.

14. Waites KB, Katz B, Schelonka RL. Mycoplasmas and ureaplasmas as neonatal pathogens. Clin Microbiol Rev. 2005;18(4):757-89. 
15. Sperling RS, Newton E, Gibbs RS. Intraamniotic infection in low-birth-weight infants. J Infect Dis. 1988;157(1): 113-7.

16. Pintucci A, Meregalli V, Colombo P, Fiorilli A. Premature rupture of membranes at term in low risk women: how long should we wait in the "latent phase"? J Perinat Med. 2014;42(2):189-96.

17. Cobo T, Kacerovsky M, Palacio M, Hornychova H, Hougaard DM, Skogstrand K, et al. A prediction model of histological chorioamnionitis and funisitis in preterm prelabor rupture of membranes: analyses of multiple proteins in the amniotic fluid. J Matern Fetal Neonatal Med. 2012;25(10):1995-2001.
18. Lahra MM, Jeffery HE. A fetal response to chorioamnionitis is associated with early survival after preterm birth. Am J Obstet Gynecol. 2004;190(1):147-51.

19. Cho HY, Jung I, Kwon J-Y, Kim SJ, Park YW, Kim Y-H. The Delta Neutrophil Index as a predictive marker of histological chorioamnionitis in patients with preterm premature rupture of membranes: A retrospective study. PLoS One. 2017;12(3):e0173382.

20. Del Pino $M$, Palacio $M$, Figueras $F$, Romans $M$, Steinvarcel F, Coll O, et al. P49. 03: Fetal Doppler parameters in pregnancies complicated with preterm premature rupture of membranes developing chorioamnionitis. Ultrasound Obstet Gynecol. 2007;30(4):640. 